\title{
Erratum to: Micafungin Elicits an Immunomodulatory Effect in Galleria mellonella and Mice
}

\author{
Beth Burgwyn Fuchs • Yan Li • Dedong Li • Tatiana Johnston • \\ Gabriel Hendricks • Gang Li • Rajmohan Rajamuthiah • Eleftherios Mylonakis
}

Published online: 2 November 2015

(C) Springer Science+Business Media Dordrecht 2015

\section{Erratum to: Mycopathologia DOI 10.1007/s11046-015-9940-z}

In the initial online publication the last name of author Tatiana Johnston was misspelled as Johnson. This has now been corrected.
The online version of the original article can be found under doi:10.1007/s11046-015-9940-z.

B. B. Fuchs $(\bowtie) \cdot$ G. Hendricks · R. Rajamuthiah · E. Mylonakis $(\square)$

Division of Infectious Diseases, Rhode Island Hospital, Alpert Medical School of Brown University, 593 Eddy Street, Aldrich 708, POB 328/330, Providence, RI, USA e-mail: helen_fuchs@brown.edu

\section{E. Mylonakis}

e-mail: emylonakis@lifespan.org

Y. Li

Pharmacy Department, Shandong Provincial Qianfoshan Hospital, Jinan, Shandong Province, China
D. Li

Department of Clinical Pharmacology, General Hospital of Chinese PLA, Beijing, China

T. Johnston

The Miriam Hospital, Alpert Medical School of Brown University, Providence, RI, USA

G. Li

Department of Laboratory Medicine, Jinshan Hospital, Shanghai Medical College, Fudan University, Shanghai, China 